El microformato informativo en los relojes inteligentes El caso de la aplicación «RTVE Informativos 24 horas»

Pavel Sidorenko Bautista, Pablo Garrido Pintado Improntas de la Historia y la Comunicación (N. ${ }^{\circ}$ 9), eo43, 2021

ISSN 2469-0457 | https://doi.org/10.24215/24690457eo43 http://perio.unlp.edu.ar/ojs/index.php/improntas FPyCS | Universidad Nacional de La Plata La Plata | Buenos Aires | Argentina

\title{
El microformato informativo en los relojes inteligentes
}

\section{El caso de la aplicación «RTVE Informativos 24 horas»}

\author{
The informative microformat in smartwatches \\ Case study of the app «RTVE Informativos 24 horas»
}

\section{Pavel Sidorenko Bautista ${ }^{1}$}

pavel.sidorenko@ufv.es

https://orcid.org/0000-0002-8094-3089

\section{Pablo Garrido Pintado ${ }^{2}$}

Pablo.garrido@ucm.es

https://orcid.org/0000-0002-6111-6715

\section{Facultad de Ciencias de la Información, Universidad Francisco de Vitoria, España 2 Universidad Complutense de Madrid, España}

\section{Resumen}

El desarrollo tecnológico ha influido en audiencias más móviles con mayor demanda de nuevos recursos que les permitan comunicarse e informarse bajo esta premisa. A través del estudio de caso del trabajo de Radio Televisión Española (RTVE), los autores analiza cómo se desarrolla el microformato informativo adaptado a los relojes inteligentes, el cual de momento se muestra como poco innovador, sin estímulos visuales y con fuerte dependencia del teléfono inteligente.

Palabras clave

periodismo, periodismo de vistazo, nuevas narrativas, relojes inteligentes

\section{Abstract}

Technological development has influenced more mobile audiences with greater demand for new resources that allow them to communicate and be informed under this premise. Through the case study of Radio Televisión Española (RTVE)'s work, the authors analyze how the news microformat adapted to smart watches is developed, which at the moment is shown as not very innovative, without visual stimuli and with a strong dependence on the smartphone.

Keywords

journalism, glance journalism, new narratives, smartwatches 


\section{El microformato informativo en los relojes inteligentes}

El caso de la aplicación «RTVE Informativos 24 horas»

\section{Por Pavel Sidorenko Bautista y Pablo Garrido Pintado}

\section{Contexto tecnológico y evolución de las narrativas periodísticas}

En 2020, de los 7750 millones de habitantes aproximadamente a nivel mundial, $67 \%$ (5190 millones) eran usuarios/as de teléfonos móviles, una cantidad superior a los 4590 millones con conexión a Internet (Hootsuite \& We Are Social, 2020). En este escenario, España supera la media de penetración mundial, con 53 millones de líneas de telefonía móvil ( $117 \%$ de la población del país) y con 43 millones de usuarios/as conectados/as a Internet ( $95 \%$ de la población total).

En este proceso, la conectividad y la movilidad han resultado variables determinantes (Silva, López \& Toural, 2017a), no solo en lo referido a la evolución de los teléfonos móviles a la tecnología wearable, ${ }^{1}$ sino también respecto de la puesta en marcha de proyectos informativos en cada una de esas plataformas que apunten a un modelo de comunicación «omnipresente» y ubicuo, ya advertido por Ramón Salaverría (2018) y por Stefanie Carlan (2019).

Se trata de un contexto en línea, multimedia e interactivo, que promueve el desarrollo constante de nuevas alternativas para construir relatos, para comunicar $y$, por ende, para informar (Herranz, Sidorenko \& Cantero, 2019), en el que las empresas periodísticas sufren una evolución orientada, en gran medida, hacia la innovación tecnológica (AnguíSánchez, Cabezuelo-Lorenzo y Sotelo-González, 2019; Nee, 2013; Serrano, 2017).

Este proceso de innovación está constituido sobre diferentes líneas estratégicas, según los casos. Desde lo meramente narrativo, mediante plataformas tan disruptivas como TikTok o el desarrollo de contenidos virtuales y en 36o- (Benítez \& Herrera, 2018; Paíno \& Rodríguez, 2019; Gutiérrez, Pérez \& López, 2020; Sidorenko, Herranz \& Cantero, 2020), frente a la necesidad de centrarse en las audiencias que no están tradicionalmente vinculadas con el consumo de noticias (Casero-Ripollés, 2020), lo cual es parte de un 
curso evolutivo del modelo mobile first (Hill \& Bradshaw, 2019) al modelo mobile only, como apunta el último estudio de Visual Capitalist sobre datos del Global Web Index (Jones, 2020). En la actualidad, las sociedades obedecen más a un comportamiento nómada y móvil (Giddens, 2003; Hjelm, 2000; Urry, 2000).

También hay una evolución de las vías de distribución de los mensajes y los contenidos. Se ha producido un salto importante y rápido de los equipos informáticos estáticos a los dispositivos móviles, que experimentan adaptaciones según el uso requerido, según las circunstancias y según el individuo. Así, en la actualidad se habla de comunicación a través de smartspeakers, smartscreens, refrigeradores o relojes inteligentes.

La sociedad está a expensas de una saturación informativa. Según Salaverría (2018), en pocos años, los individuos han pasado de buscar noticias a sentirse desbordados por estas y por demás informaciones. El mundo ha pasado del poder de los soportes a las audiencias y de la exposición a la acción, materializada en la integración del social media y en la proliferación de sistemas de recomendación. El contenido se desliga del soporte y el consumo es multipantalla (Castellet, Aguado \& Martínez, 2013), lo que da paso al advenimiento de narrativas transmedia.

En cuanto a los dispositivos de acceso, año a año se comprueba que el smartphone continúa siendo el dispositivo de referencia para la gestión de la vida digital. Según el informe Ditrendia (2019), 96\% de los/as usuarios/as que accede a Internet a diario utiliza para ello su smartphone, lo que resulta revalidado por el estudio de consumo digital realizado por Visual Capitalist sobre datos del Global Web Index durante el primer cuatrimestre de 2020, en el marco del inicio de la pandemia por Covid-19 (Jones, 2020).

Específicamente, en relación con el acceso a medios informativos, en 2020, el Digital News Report elaborado por Reuters (Newman y otros, 2020) señala un incremento en el consumo informativo a través de servicios de mensajería instantánea como WhatsApp y grupos de la red social Facebook. Aunque la crisis sanitaria provocada por el Covid-19 ha producido un aumento del consumo de información a través de medios tradicionales, los digitales se mantienen como la principal vía. Mientras tanto, las audiencias más jóvenes han buscado la información en Instagram, Snapchat o TikTok (Sidorenko, Herranz \& Cantero, 2020).

Sin embargo, otros dispositivos móviles empiezan a tener protagonismo. Los hábitos de consumo informativo y de noticias de los españoles se ubican: 67\% desde los smartphones, $46 \%$ desde los ordenadores, $21 \%$ a través de aplicaciones de televisores 
inteligentes, $20 \%$ desde otros dispositivos móviles como tablets, $2 \%$ desde altavoces inteligentes y, apenas, $1 \%$ desde los relojes inteligentes (Newman y otros, 2020), siendo este último indicador muy bajo si se toma como referencia que $21 \%$ de usuarios/as de Internet en España cuenta con un dispositivo de estas características (IAB Spain, 2020).

Es fácil presuponer que, de momento, su funcionalidad en términos generales se centre más en la réplica de notificaciones desde el smartphone, para escuchar música (los modelos con conexión móvil LTE y conexión bluetooth para auriculares) y, principalmente, para monitorear la actividad física, pues todos los modelos cuentan, en general, con un amplio abanico de opciones de registro de ejercicios, de hábitos de sueño, de frecuencia cardíaca e, inclusive, de oxígeno en sangre.

La acepción actual de estos dispositivos se puede identificar a partir de 2012, cuando Pebble, Samsung y Apple comenzaron a pugnar por el mercado de este tipo de dispositivos (De Sousa, 2017), interconectados inicialmente a los smartphones, pero que paulatinamente han ido ganando autonomía.

Por sus características, autores como Yoonhyuk Jung, Kim Seongcheol y Boreum Choi (2016) apuntan a la naturaleza híbrida, entre un dispositivo informático y un accesorio de moda. Capacidades que han aumentado considerablemente con las funciones de comunicación por voz, que le otorgan al dispositivo mayor independencia frente a otros dispositivos móviles, aunque, en muchos modelos, el tamaño de la pantalla y la conexión indirecta a smartphones aún es un gran inconveniente.

Los relojes inteligentes -especialmente, los fabricados por Samsung hasta el momento-, son dispositivos que integran diversos elementos interactivos como servicios de mensajería de texto, llamadas por voz, administración de archivos y de contenidos multimedia, herramientas para la optimización de la productividad e, inclusive, aplicaciones para el ocio, a partir de un pequeño dispositivo para ser dispuesto en la muñeca de las personas y que debe estar emparejado con un teléfono inteligente, con el fin de obtener el máximo provecho de las posibilidades que se ofrecen (Jackson, 2019).

\section{El «periodismo de vistazo» en los relojes inteligentes}

Dada su cercanía con los/as usuarios/as, los relojes inteligentes representan una solución interesante para la difusión de productos periodísticos, al procurar una distribución de contenidos y de información más rápida que el propio smartphone (Silva, López y Toural, 
2017b). En otras palabras, a través de los relojes inteligentes la información puede llegar a las personas de forma más precisa, por tratarse de dispositivos que están a disposición más rápida, con pantalla y con opciones suficientes para permitir, por ejemplo, un periodismo de titulares a priori.

Según Paul Bradshaw (2017), con la llegada del Apple Watch se acuñó la expresión «glance journalism» o periodismo de vistazo, gracias a la característica que le permitía a los/as usuarios/as leer las notificaciones en este dispositivo. Así, diversos medios han diseñado aplicaciones centradas en el glance-optimised headline (GOH), expresión que en español puede ser traducida como «título optimizado a la mirada». Este tipo de notificaciones, que aparecen en la pantalla de manera fugaz, fomentan lo que en términos de búsqueda se denomina «click-zero». Es decir, el/la usuario/a obtiene de un vistazo una breve píldora de información sin ejecutar ninguna acción posterior.

Para Sarah Marshall (2014), el auge del periodismo de vistazo viene heredado del predominio de Twitter y responde a una necesidad de los medios de estar en todo tipo de plataformas. Frente a esto, la autora plantea la siguiente pregunta: si todo contenido es gratuito, ¿quién paga los salarios de comunicadores/as y de periodistas? La visión de Marshall (2014) no es tan negativa: «Si atendemos a audiencias en diferentes plataformas (vistazo a un reloj o notificaciones push) las personas acudirán a nosotros cuando necesiten la historia completa, mucho más allá de lo que un simple vistazo puede proporcionar» (Traducción de los autores del artículo). ${ }^{2}$

Ahora bien, estudios como el de Bilge Narin (2018) señalan, también, los efectos negativos de la popularización de este modo de difundir noticias. Para la autora, tanto los fabricantes tecnológicos como los medios han promocionado el nuevo tipo de periodismo con la idea de «menos es más». Sin embargo, con base en las teorías de Jacques Ellul (1986), los relojes inteligentes pueden representar una desventaja en la calidad y en la capacidad semántica de las noticias, es decir, el periodismo de vistazo conlleva el riesgo de fomentar el discurso dominante y de sacrificar el periodismo de calidad.

En una posición intermedia, Logan Molyneux (2018) alude en sus reflexiones a dos posturas. Por una parte, destaca a quienes defienden el consumo de noticias a través de móviles, lo que implica tener que dedicar un tiempo limitado a las noticias en lugar de desarrollar un aprendizaje más profundo sobre los asuntos públicos. Por otra, la existencia, en contraste, de patrones de consumo complejos y multifacéticos, donde se 
alternan momentos de consumo rápido, mientras estamos en movimiento, con lecturas más largas, en el sofá o en la cama por la noche. Así, las audiencias móviles tienden a usar sus dispositivos en múltiples ocasiones a lo largo del día, por lo que el tiempo que le dedican a las noticias es elevado (Westlund, 2012).

\section{RTVE y el desarrollo del periodismo para dispositivos móviles}

En la actualidad, son muchas las aplicaciones de noticias para los diferentes ecosistemas de relojes inteligentes. Tomando como referencia a los dos fabricantes con mayor cuota de mercado a nivel global, en 2019, en los dispositivos Apple era posible encontrar, por ejemplo, Flipboard (agregador de noticias), The Wall Street Journal, CNN, El País, Euronews, Le Monde, CGTN y RT, mientras que en los dispositivos Samsung se podía acceder a Flipbord, Upday (agregador de noticias exclusivo de Samsung), News Republic (agregador de noticias de la tecnológica china ByteDance), De Telegraaf, RTVE Río 2016 y RTVE informativos 24 horas (Fernández, 2020).

Todos estos medios y servicios noticiosos tienen, por igual, sus respectivas aplicaciones para smartphones y tablets. De hecho, hay más desarrollo de aplicaciones móviles por parte de las empresas periodísticas para estas dos últimas tipologías de dispositivos que para los relojes inteligentes, donde la propuesta aún es incipiente.

Frente a esto, la corporación pública española de medios Radio Televisión Española (RTVE) ha venido adelantando un trabajo importante, al crear diferentes productos informativos dedicados a los dispositivos móviles. Así, para smartphones y tablets, bajo la denominación «RTVE Medios Interactivos», ha desarrollado con carácter periodístico: RNE, RTVE Noticias, Radio 3, El Tiempo de RTVE, TDP, RTVE VR 36o, y Vive Río: Heroínas JJOO en VR, a las que vale sumar, exclusivamente para los relojes inteligentes de Samsung, las antes referenciadas RTVE Río 2016 y RTVE informativos 24 horas.

\section{Metodología}

El desarrollo del formato y de los contenidos adaptados a los relojes inteligentes aún es embrionario. Los medios de comunicación no han estandarizado su oferta de contenidos, pese a que ya existe un amplio desarrollo de sistemas operativos y de productos. En este sentido, se observa cómo mientras unos apuestan por los dispositivos de la estadounidense 
Apple, con sistema Watch OS, otros se decantan por la propuesta de los dispositivos de Samsung, con su sistema Tizen, por nombrar solo los más representativos y con mayor penetración en el mercado de este tipo de dispositivos. ${ }^{3}$

En otras palabras, si bien el ecosistema de interfaces y de entornos operativos de los relojes inteligentes es variado, el de medios con sus aplicaciones adaptadas a estos dispositivos es mucho más heterogéneo, hecho que se contrapone a lo que ocurre en relación con dispositivos móviles como los smartphones y las tablets, donde es común ver las mismas aplicaciones en dispositivos con distintos sistemas operativos. En los relojes inteligentes, esta desigual distribución de aplicaciones supone un problema para llegar a la mayor audiencia posible.

Para el presente trabajo, se propone analizar el caso de la aplicación de noticias «RTVE informativos 24 horas», del medio público español RTVE -disponible, únicamente, en los relojes inteligentes de Samsung con sistema operativo Tizen-, la cual tiene una valoración en la tienda de aplicaciones de la empresa surcoreana Galaxy Store de 4.o sobre 5.o. Se tomó en consideración este caso debido a que se trata de una aplicación que funciona en el reloj de manera autónoma, sin necesidad de tener una aplicación de soporte instalada en el teléfono inteligente, como ocurre con agregadores de noticias como Upday, Flipboard o News Republic, en Samsung, o El País y CNN, en Apple, en los que solo se presenta un esbozo de la información, la cual se extiende en el smartphone a través del botón «continuar en el teléfono». En estos casos, el reloj inteligente funciona como una breve extensión del smartphone.

La observación se realizó durante las dos primeras semanas del mes de junio de 2020 a través de un Samsung Galaxy Watch, con la finalidad de comprobar la actualización de la información publicada. Los contenidos, en tanto, se contrastaron con los de la aplicación para dispositivos móviles «RTVE noticias» y con la web https://www.rtve.es/noticias, para realizar las comprobaciones propuestas y para evidenciar hasta qué punto la opción del reloj inteligente constituye un elemento diferenciador al momento de hacer frente a la segmentación de audiencias, llevándose a cabo una debida adaptación del dispositivo y del formato en cuestión.

La primera semana ( 1 al 7 de junio de 2020) se reservó para observar la periodicidad en las actualizaciones de contenidos; la segunda ( 8 al 14 de junio de 2020), para llevar a cabo, de manera aleatoria, las comparaciones entre el contenido de la aplicación para 
relojes inteligentes, la versión para dispositivos móviles del tipo smartphone y tablet, y la versión web.

Para los fines del estudio, se plantearon varias hipótesis de partida. En primer lugar, se presume que el objeto de estudio se adapta al concepto y a la dinámica de «periodismo de vistazo» (Bradshaw, 2017; Silva, López \& Toural, 2017b) en el microformato que implica la pantalla de 1.3 pulgadas del equipo $\left(\mathrm{H}_{1}\right)$. Asimismo, entendiendo el trabajo que demanda el desarrollo y la programación de aplicaciones móviles, se considera que representa un elemento clave en la estrategia multidispositivo que lleva a cabo la corporación pública de medios RTVE $\left(\mathrm{H}_{2}\right)$. Por último, se parte de la idea de que la aplicación en cuestión constituye una oferta informativa personalizada y adaptada, dada la particular cercanía del dispositivo con al/la usuario/a $\left(\mathrm{H}_{3}\right)$.

El objetivo principal de la investigación ha sido comprobar hasta qué punto el medio seleccionado para este estudio trabaja en experiencias particulares en su estrategia multidispositivo, en este caso específico, respecto del desarrollo de una narrativa informativa en microformato para relojes inteligentes.

\section{Análisis de resultados}

$\mathrm{Al}$ ejecutar en el reloj inteligente la aplicación «RTVE informativos 24 horas», se presentan dos opciones: «En portada» $\mathrm{y}$ «Secciones».

En el caso de la primera, la aplicación permite disponer de doce noticias que resumen la actualidad de las secciones y los temas abordados por el medio. Se trata de doce informaciones seleccionadas del conjunto inicial, que también se puede apreciar en la aplicación móvil y en la web. En la versión para relojes inteligentes, este número nunca varía, a diferencia de lo que sucede con las otras plataformas.

La experiencia de usuario es muy simple: si se selecciona «En portada» [Figura 1, núm. 2], se muestra el primer titular con media imagen referencial y un indicador de número de contenido en la parte superior de la ventana, simbolizado por pequeños puntos [Figura 1, núm. 5]. Esta es la interfaz estándar de todas las publicaciones en todas las secciones.

Como se aprecia en la misma imagen, la gráfica está acompañada del titular que, si se pulsa, permite derivar al artículo completo [Figura 1, núm. 6]. 


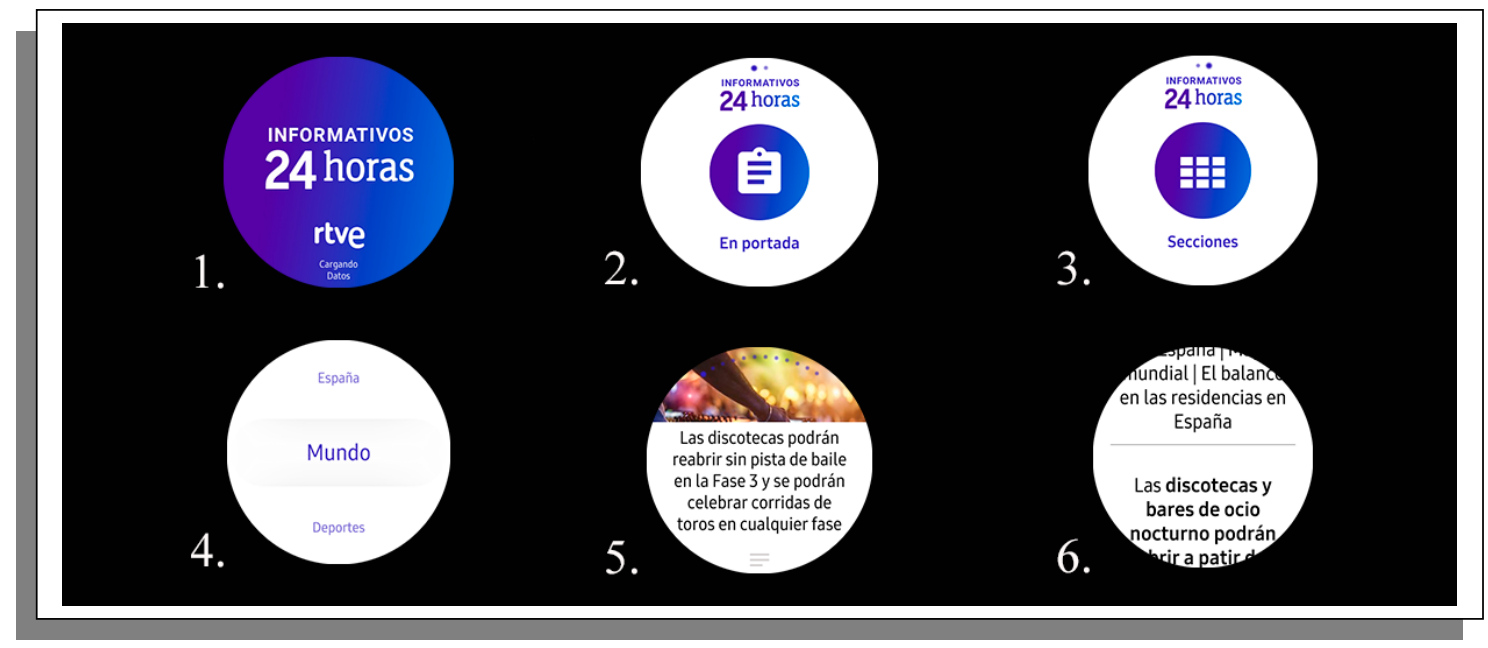

Figura 1. Arquitectura de la aplicación «RTVE Informativos 24 horas». Captura de pantalla tomada el 6 de junio de 2020. Fuente: elaboración propia

Como se advirtió, RTVE presenta un amplio desarrollo de contenidos para todas las plataformas digitales y para todos los dispositivos interconectados, lo que incluye los asistentes virtuales por voz y vídeo, tanto en Google Home como en Alexa, así como en plataformas virtuales como Oculus. ${ }^{4}$

En este sentido, se procedió a contrastar los contenidos de «RTVE informativos 24 horas» con los de la aplicación para dispositivos móviles Android e iOS «RTVE noticias» y con la sección noticias del sitio del mismo medio [Figura 2], por ser las otras tribunas digitales que manejan la misma clase de contenidos que la aplicación aquí estudiada y que tienen un alto alcance de audiencia después de la televisión.

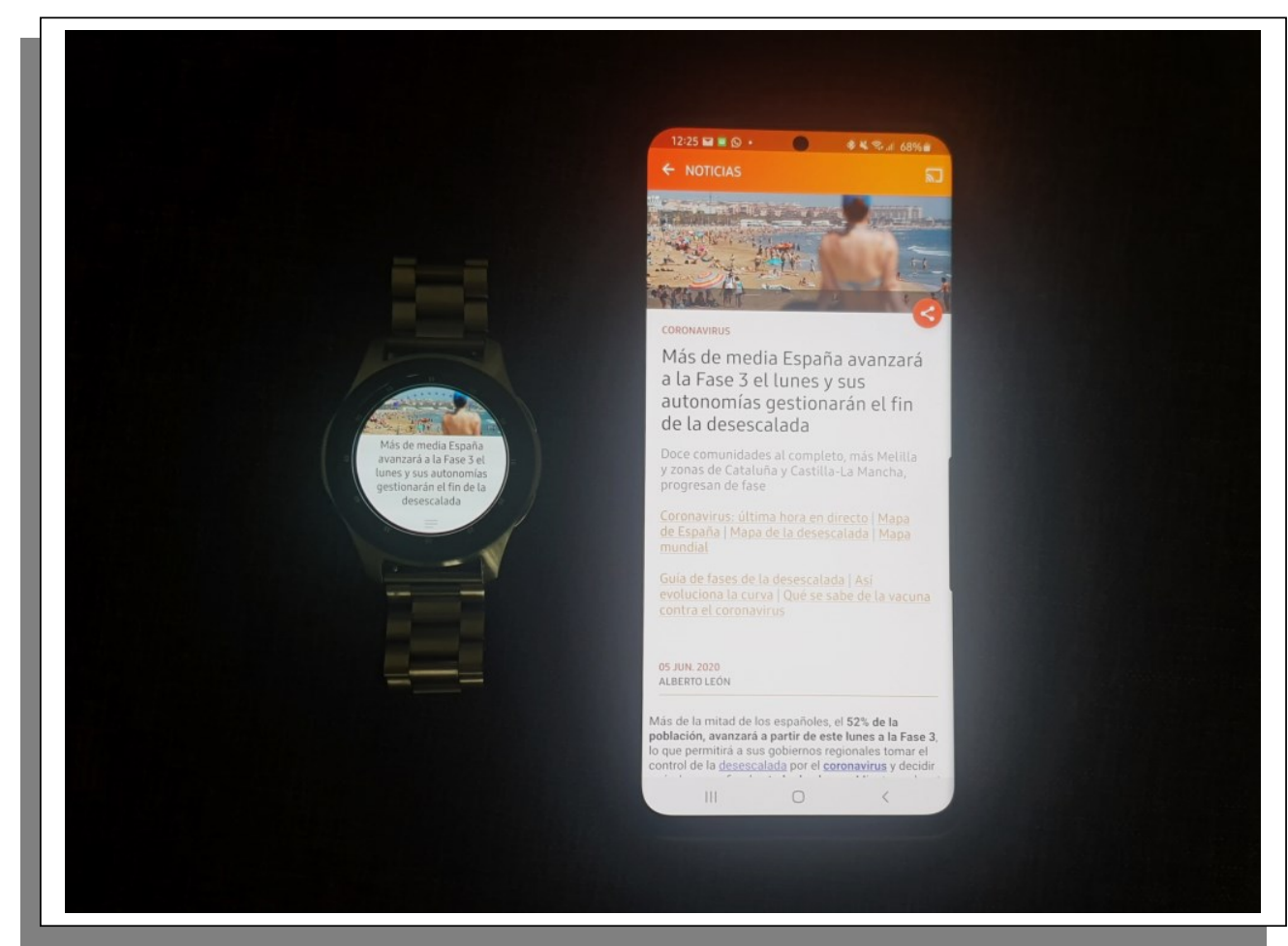

Figura 2. Contenido en las aplicaciones «RTVE Informativos 24 horas» $\mathrm{y} «$ RTVE Noticias». Fuente: elaboración propia 
Como se aprecia, el recurso gráfico es el mismo, así como el titular de la noticia [Figura 2]. Cabe destacar que a diferencia de la aplicación para smartphones y para tablets, en la versión para relojes inteligentes no hay notificaciones «push», es decir, para estar al tanto de la actualidad el/la usuario/a debe acudir directamente a la aplicación y dirigirse o bien a la sección de portada, que presenta un resumen de todos los temas, o a una sección en particular, lo cual es una experiencia de usuario/a muy similar a la de otras aplicaciones de medios como «BBC News» para relojes con sistema Wear OS (De Sousa, 2017).

Quizás lo más llamativo ocurre cuando la comparación se extiende al contenido del sitio web. En la Figura 3 se aprecia que la noticia cuyo titular reza «La Abogacía del Estado acusa a la jueza del 8M de iniciar una búsqueda voraz de delito contra el Gobierno», publicada a primera hora del 9 de junio 2020, se replica exactamente igual en el reloj inteligente, en el smartphone y en el ordenador.

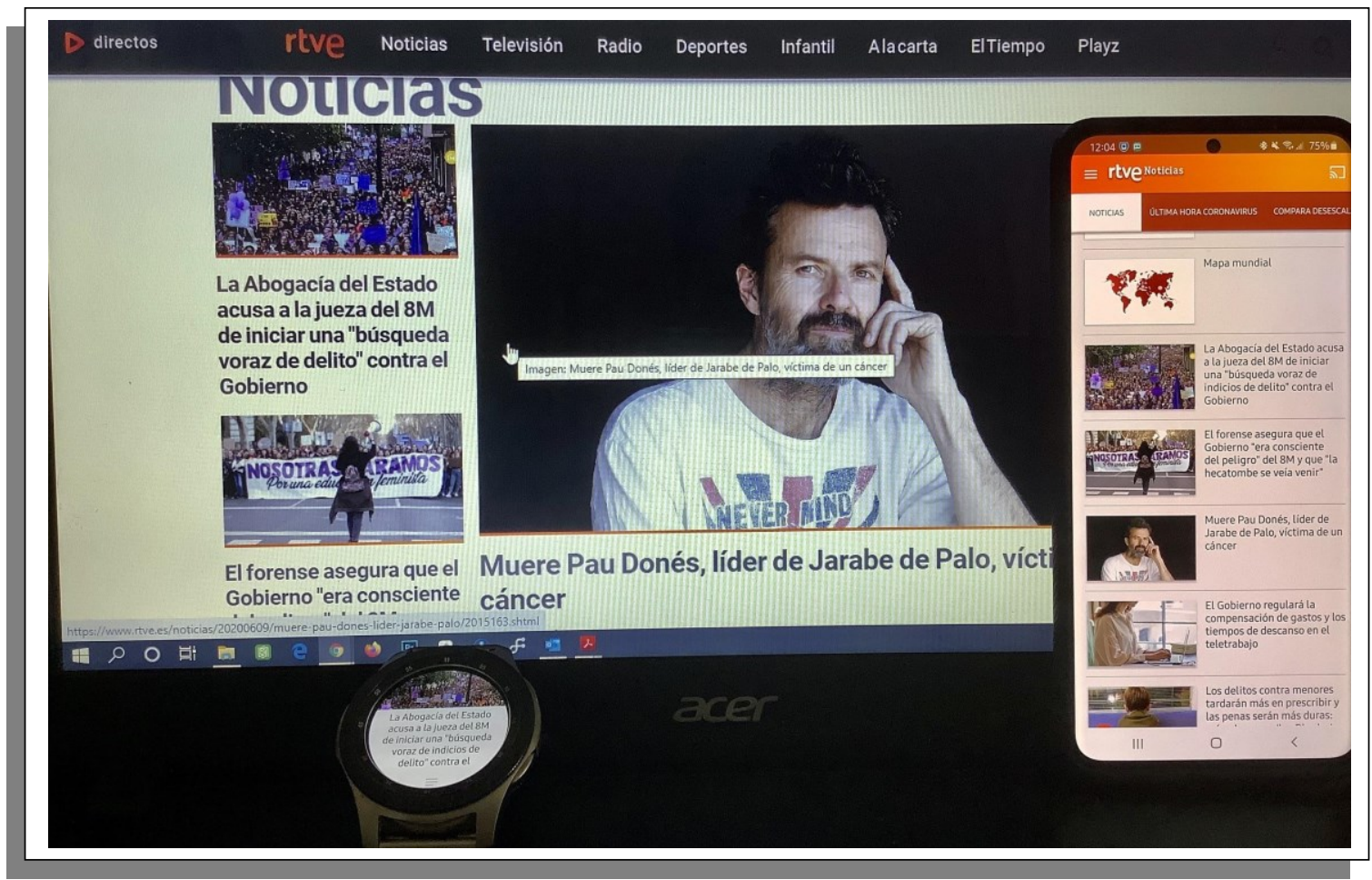

Figura 3. Presentación de igual contenido en el sitio de RTVE Noticias, y en las aplicaciones «RTVE Informativos 24 horas» $y$ «RTVE Noticias».

Fuente: elaboración propia

Esto obligó a indagar más y a revisar el texto en las tres interfaces, a fin de constatar si se trataba del mismo relato o si había sido modificado en función de la pantalla de consumo, entendiendo las particularidades del microformato en los relojes inteligentes. 
El análisis permite afirmar que no existe variación alguna con el texto, a excepción de los recursos gráficos y del hipertexto, que en la versión para relojes inteligentes no existe. De hecho, las frases o las palabras con enlaces activos en el Galaxy Watch aparecen simplemente con negritas.
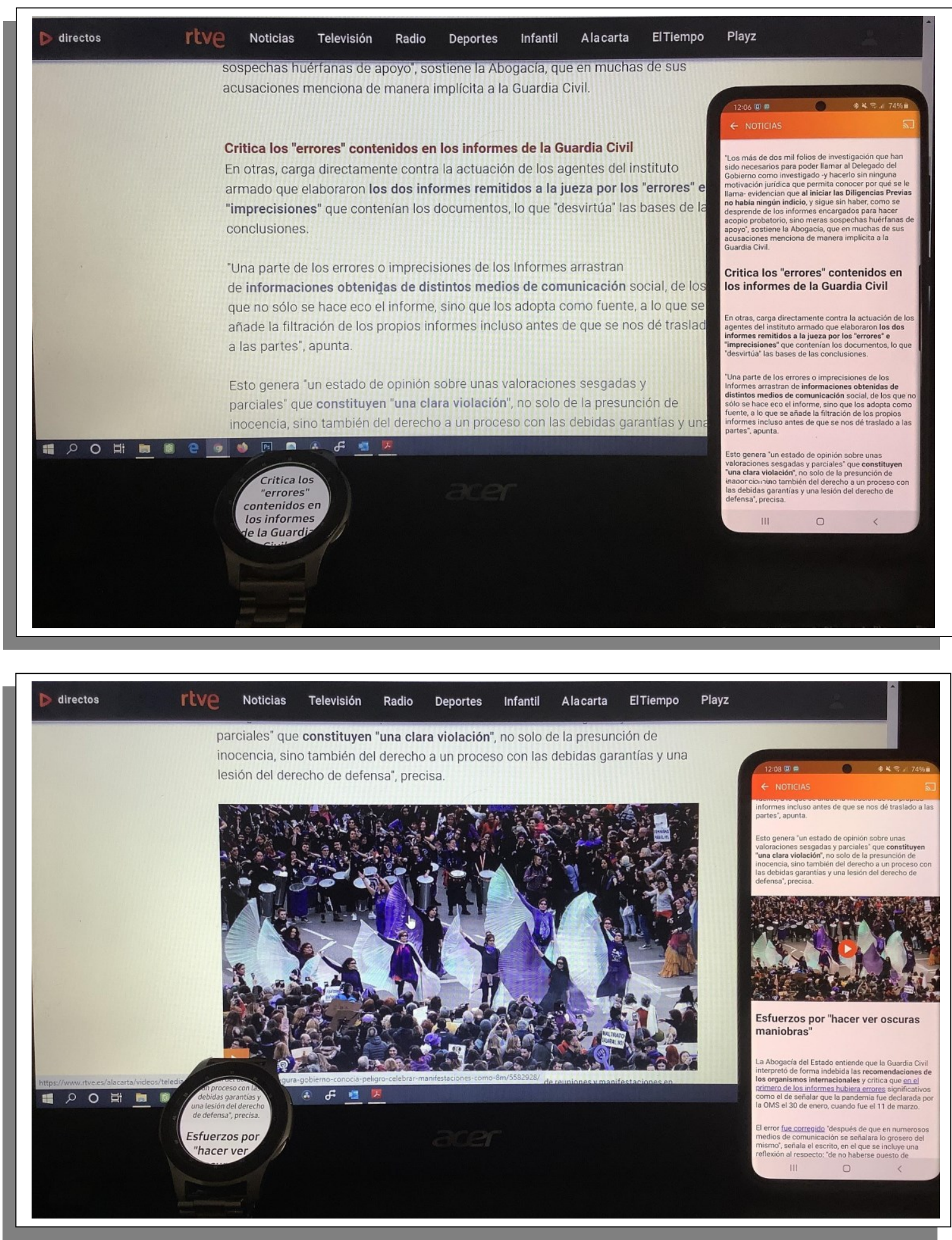

Figuras 4 y 5 . Desarrollo de igual contenido en el sitio de RTVE Noticias y en las aplicaciones «RTVE Informativos 24 horas» $y$ «RTVE Noticias». Fuente: elaboración propia 
El ritmo de actualización de las noticias en la aplicación del reloj inteligente es proporcional al de la aplicación para smartphones y al del sitio web. En líneas generales, durante el segundo período de análisis se observaron, en promedio, tres actualizaciones a lo largo del día. Vale aclarar que, pese a este proceso, algunas noticias prevalecían durante todo el día.

Por tanto, si bien es cierto que el texto está perfectamente adaptado a la particular pantalla circular de 1.3 pulgadas de diámetro del reloj en cuestión, y que la experiencia de usuario/a implica que con un suave desplazamiento vertical sea posible avanzar o retroceder en la lectura, también es cierto que por parte del medio no hay una intención por desarrollar una experiencia particular, pues en todas las interfaces se replica el mismo trabajo.

En este caso, únicamente se prescinde de los recursos hipertextuales y multimedia, que entorpecerían la experiencia dentro de la aplicación. Un aspecto técnico que se pudo apreciar mediante la observación de las otras aplicaciones de noticias aludidas, para el caso del dispositivo aquí sometido a estudio, las cuales ninguna utiliza estos recursos interactivos.

\section{Conclusiones}

El ecosistema de relojes inteligentes es cada vez más grande y más diverso. Como ocurre con otros dispositivos móviles, las pantallas de estos dispositivos varían en la forma (actualmente, entre cuadrada o circular) y en las dimensiones, lo cual puede suponer una complejidad a la hora de desarrollar una propuesta digital en microformato. Presumiblemente, esto se relacione con que en este momento los/as productores/as de contenidos ponderen la opción de una tecnológica en particular para desarrollar su propuesta, en lugar de intentar llevar a esta última a la mayor cantidad de dispositivos posibles como ocurre con los smartphones y las tablets.

En el caso particular de RTVE, lo primero que llama la atención es que mientras el apartado web y la aplicación para smartphones y tablets se denomina «RTVE noticias», la aplicación móvil para los relojes inteligentes con sistema operativo Tizen (Samsung) recibe el nombre de «RTVE informativos 24 horas», lo cual en primera instancia hace pensar que se trata de experiencias informativas diferenciadas, cuando no es así. 
De esta manera, si se revisa el caso de estudio en relación con la primera hipótesis, referida a la adaptación del trabajo de RTVE para los relojes inteligentes de Samsung con respecto al concepto y a la dinámica de «periodismo de vistazo» (Silva, López \& Toural, 2017b), se puede afirmar que pese a que el periodismo «de vistazo» implica el consumo rápido de un contenido informativo -planteado, cada vez más, desde el modelo de ubicuidad referido por Carlan (2019) y por Salaverría (2018)-, en este particular no se aprecia, ya que al prescindirse del recurso de la notificación «push», el/la usuario/a se ve obligado/a a acudir a la aplicación para acceder al contenido periodístico, en ocasiones, a destiempo en relación con la actualidad informativa.

Sin embargo, una vez dentro de la aplicación, e independientemente de la sección escogida [Figura 1, núm. 4], el sistema introductorio de imagen y de titular, y una selección de doce o de menos contenidos, sí se propone una navegación que se aproxima a la propuesta de «visualización exprés»; no obstante, el titular ofrece una referencia informativa excesivamente limitada.

Asimismo, el hecho de que la aplicación del reloj funcione de manera autónoma supone una ventaja sobre la de otros medios como BBC y Le Monde (De Sousa, 2017) o de los agregadores de noticias referenciados (Upday, Flipboard y News Republic), que no permiten consumir la noticia desarrollada si no se tiene acceso al smartphone con su respectiva aplicación, también instalada.

En este sentido, tampoco se cumple la segunda hipótesis, ya que si se cuenta con la aplicación del smartphone -como es el caso de quienes suscriben el presente estudio-, la opción del reloj carece de sentido, pues la experiencia de usuario/a es mucho más completa en la primera opción, que permite interactuar con elementos multimedia e hipertextuales. A esto, debe añadirse que para gozar de conexión a la red, desde este modelo de reloj en particular, el teléfono debe estar a una distancia no superior a los tres metros, pues están conectados vía bluetooth y es el smartphone el proveedor de conexión a la red de datos móviles. Vale agregar, además, que como estrategia de penetración en el ámbito de los wearables esta modalidad es limitada, ya que solo está disponible en el ecosistema de Samsung.

Por último, en cuanto a la tercera hipótesis, referida a que si el caso de estudio constituye una oferta informativa personalizada y adaptada al/la usuario/a, dada la particular cercanía del dispositivo con este/a, se puede afirmar que no se cumple por las razones ya expuestas: aunque la aplicación es autónoma, se requiere del smartphone para 
proporcionar conexión de red. Asimismo, la estrategia emprendida hasta este momento por el medio en cuestión supone una réplica de sus publicaciones en el sitio web, tanto en la aplicación para smartphones como para relojes inteligentes, inclusive, con menos elementos interactivos en el segundo caso. La aplicación no admite que el/la usuario/a escoja los temas que quiere seguir y sobre los que quiere profundizar, como sí ocurre con otras aplicaciones informativas.

La audiencia tampoco tiene la posibilidad de filtrar o de escoger los temas de interés. No obstante, se trata, sin duda, de un precedente importante que marca la pauta sobre la necesidad que tienen los/as productores/as de contenidos y, en especial, los medios de comunicación y los/as periodistas de adaptarse a las narrativas y a las interfaces que van desarrollándose como consecuencia de la acelerada evolución tecnológica. Este proceso ha supuesto una segmentación cada vez mayor de las audiencias, que se agrupan en nichos digitales específicos, por lo que, si la pretensión desde el periodismo es hacer llegar la información a la mayor cantidad de usuarios/as posible, el esfuerzo debe ser mayor en este sentido.

Una gran debilidad que se observa en este caso es la ausencia de notificaciones «push», que apostaran más por el periodismo de vistazo, y una mayor polivalencia de la noticia en microformato como ocurre, por ejemplo, con la aplicación del diario El País en los dispositivos Apple Watch, donde se producen alertas y notificaciones en forma de titular, con la salvedad de que la aplicación en cuestión no es autónoma y requiere de la operacionalidad del reloj inteligente. Sin embargo, este sistema de notificaciones apunta más al modelo periodístico ubicuo, vaticinado y referido como el futuro inmediato por Carlan (2019) y por Salaverría (2018).

Sin duda, el desarrollo de los microformatos periodísticos tiene un extenso camino por transitar, en especial, en un segmento de productos que goza de una cada vez mayor penetración entre los/as usuarios/as, en la línea de dispositivos independientes, más que como una simple extensión del smartphone, tal como está planteado de momento.

Aún queda mucho por desarrollar en cuanto a interfaces y modelos de interacción con el usuario. Los relojes inteligentes resultan elementos digitales más cercanos al/la usuario/a que el propio smartphone, y sobre ello se debe pensar en el futuro inmediato a la hora de desarrollar productos periodísticos y de comunicación. 
El microformato debe trascender la simple idea de adaptarse a las especificaciones de este nuevo estilo de pantalla, permitiendo mayor interactividad y autonomía de los contenidos, características que no se observaron en el presente estudio. Inclusive, si se atiende al hecho de que los hábitos de consumo de los individuos son diferentes, en algún momento debería brindarse a la audiencia la posibilidad de personalizar la experiencia multicanal del medio escogido y de señalar el tipo de información que desea recibir.

\section{Referencias}

Anguí-Sánchez, D., Cabezuelo-Lorenzo, F. y Sotelo-González, Jo. (2019). Innovación tecnológica y social en los nuevos medios digitales norteamericanos. Análisis del caso de Quartz News. Revista Latina de Comunicación Social, (74), 1697-1713.

https://doi.org/10.4185/RLCS-2019-1405

Benítez de Gracia, M. J. y Herrera Damas, S. (2018). Los primeros pasos del reportaje inmersivo a través de vídeos en 360‥ Historia y Comunicación Social, 23(2), 547-566.

http://dx.doi.org/10.5209/HICS.62784

Bradshaw, P. (2017). The Online Journalism Handbook. Skills to Survive and Thrive in the Digital Age. Birmingham, England: Routledge.

Carlan, S. (2019). Jornalismo ubíquo para smartphones: proposta de instrumento de análise de propiedades técnicas. En J. Canavilhas, C. Rodrigues, y F. Giacomelli (Eds.), Narrativas jornalísticas para dispositivos móveis (pp. 57-76). Covilha, Portugal: Labcom.IFP.

Casero-Ripollés, A. (2020). Impact of Covid-19 on the Media System. Communicative and Democratic Consequences of News Consumption during the Outbreak. El profesional de la información, 29(2), e290223. https://doi.org/10.3145/epi.2020.mar.23

Castellet, A., Aguado, J. M. y Martínez, I. (2013). Los nuevos actores que cambian las reglas y el juego. Cómo Apple y Google han transformado la distribución de contenidos. En K. Zilles, J. Cuenca y J. Rom (Coords.), Breaking the Media Value Chain. VII International Conference on Communication and Reality (pp. 333-342).

Recuperado de https://bit.ly/3BECkbz 
De Sousa, M. E. (2017). Notícias no pulso: Uma análise de aplicativos jornalísticos em relógios inteligentes. Revista Observatório, 3(1), 457-479.

https://doi.org/10.20873/uft.2447-4266.2017v3n1p457

Ditrendia (2019). Mobile en España y el mundo 2019 [Informe].

Recuperado de https://mktefa.ditrendia.es/informe-mobile-2019

Ellul, J. (1986). La palabra humillada. Madrid, España: General Tabanera.

Fernández, R. (2020). Cuota de mercado mundial de los fabricantes de smartwatches 2017-2019. Statista. Recuperado de http://bit.ly/3rsJ6Mh

Giddens, A. (2003). Runaway World. How Globalisation is Reshaping Our Lives.

New York, United States: Routledge.

Gutiérrez, B., Pérez, S. y López, X. (2020). Las secciones y Apps de RV y vídeos en 360º a examen. Estudio de caso de siete medios con impacto en Europa. Revista Latina de Comunicación Social, (75), 149-167. https://www.doi.org/10.4185/RLCS-2020-1420

Herranz, J. M., Sidorenko, P. y Cantero, J. I. (2019). Realidad virtual y periodismo inmersivo: nuevas formas de contar historias para los periodistas. En M. J. Ufarte

(Coord.), Nuevos perfiles profesionales para el mercado periodístico (pp. 127-148).

Salamanca, España: Comunicación Social.

Hill, S. y Bradshaw, P. (2019). Mobile-first Journalism. London, England: Routledge.

Hjelm, J. (2000). Designing Wireless Information Services. Boston, United States:

John Wiley \& Sons.

Hootsuite y We Are Social (2020). Digital 2020. Global Digital Overview.

Recuperado de https://bit.ly/3oxbLDe

IAB Spain (2020). Estudio de Redes Sociales 2020. Recuperado de https://bit.ly/2DYfTF6

Jung, Y., Seongcheol, K. y Choi, B. (2016). Consumer Valuation of the Wearables.

The Case of Smartwatches. Computers in Human Behavior, (63), 899-905.

https://doi.org/10.1016/j.chb.2016.06.040

Jackson, W. (2019). SmartWatch Design Fundamentals. WatchFace Design for Samsung Galaxy SmartWatches. Berkeley, United States: University of California. 
Jones, K. (7 de abril de 2020). How COVID-19 Has Impacted Media Consumption, by Generation. Visual Capitalist. Recuperado de https://www.visualcapitalist.com/media-consumption-covid-19/

Marshall, S. (5 de octubre de 2014). Glance Journalism: News without a Click [Entrada de blog]. Recuperado de https://bit.ly/32Mb2RF

Molyneux, L. (2018). Mobile News Consumption. A Habit of Snacking.

Digital Journalism, 6(5), 634-650.

Narin, B. (2018). Less is More or Less is Biased: Glance Journalism [Conference].

In 16th International Symposium Communication in the Millennium (92-99).

Association of Turkish and American Communication Scholars.

Nee, R. (2013). Creative Destruction: an Exploratory Study of How Digitally Native News Nonprofits are Innovating Online Journalism Practices. International Journal on Media Management, 15(1), 3-22.

Newman, N., Fletcher, R., Schulz, A., Simge, A. y Nielsen, R. K. (2020). Reuters Institute. Digital News Report 2020. Recuperado de https://bit.ly/3fVeIUD

Paíno, A. y Rodríguez, M. I. (2019). Propuesta de «géneros periodísticos inmersivos» basados en la realidad virtual y el vídeo 36o. Revista Latina de Comunicación Social, (74), 1132-1153. http://dx.doi.org/10.4185/RLCS-2019-1375

Salaverría, R. (16 de enero de 2018). Allá donde estés habrá noticias. Cuadernos de periodistas, (35), 15-22. Recuperado de https://www.cuadernosdeperiodistas.com/alladonde-estes-habra-noticias/

Serrano, P. (2017). La transformación digital de una redacción y el periodismo móvil (MOJO). Barcelona, España: UOC.

Sidorenko, P., Herranz, J. M. y Cantero, J. I. (2020). Use of New Narratives for COVID-19 Reporting. From 36o - Videos to Ephemeral Tiktok Videos in Online Media. Trípodos, 1(47), 105-122. https://doi.org/10.51698/tripodos.2020.47p105-122

Silva, A., López, X. y Toural, C. (2017a). A Review of Mobile Journalism in Spain. En Á. Rocha y otros (Eds.), Recent Advances in Information Systems and Technology, Advances in Intelligent Systems and Computing 571 (pp. 93-100). New York, United States: Springer. 
Silva, A., López, X. y Toural, C. (2017b). Los iWatch: el intenso flujo de microformatos de «periodismo de un vistazo» alimentan seis de los principales medios online. Revista Latina de Comunicación Social, (72), 186-196. http://dx.doi.org/10.4185/RLCS-2017-1160

Urry, J. (2000). Sociology beyond Societies. Mobilities for the Twenty-first Century. New York, United States: Routledge.

Westlund, O. (2012). Mobile News. A Review and Model of Journalism in an Age of Mobile Media. Digital journalism, 1(1), 6-26. Recuperado de https://www.tandfonline.com/doi/full/10.1080/21670811.2012.740273

Notas

1 Traducido al español como «usable», se refiere a diferentes artefactos utilitarios interconectados.

2 «My view is that if we serve audiences on different platforms -whether that be via a glance on a watch, in a Twitter feed, as a push notification or via Google Glass- people will come to us when they need the full story, way beyond what the glance can provide» (Marshall, 2014).

3 En el estudio publicado en Statista (Fernández, 2020), se hace referencia a un 32\% de la cuota de mercado representada por «Otros». Desde China, las empresas Xiaomi y Huawei vienen desarrollando actualmente un amplio catálogo de productos al respecto, seguidas por compañías como Honor, Oppo y Realme, por referenciar algunas. Sin embargo, por la particularidad de los sistemas de estos equipos -aunque no por sus precios, que son más competitivos-, el catálogo de aplicaciones y, por ende, la comunidad de desarrolladores/as, es mucho mayor en relación con los tres sistemas aludidos.

4 Véase https://www.rtve.es/rtve/voz/ y https://ocul.us/2AGfcPd 